\title{
铜催化异氭酸酯加成反应机理研究
}

\author{
黄 利 $a$ 王毓浩 $a$ 刘吉英 $a$ 李世俊 $*, a$ 张文静*, $a$ 蓝 宇*,a,b \\ ( ${ }^{a}$ 郑州大学化学学院 绿色催化中心 郑州 450001) \\ ( $b$ 重庆大学化学化工学院 理论与计算化学重庆市重点实验室 重庆 400001)
}

\begin{abstract}
摘要 异氰酸酯作为一种重要的单碳碳源, 在合成化学中可以用于制备酰胺衍生物或杂环类化合物, 其活化和转化机 制是一个重要的问题. 通过密度泛函理论(DFT)计算研究了亚铜催化异氰酸酯氢嗍乙基化反应的机理. 研究结果表明, 该反应中叔丁醇亚铜是活性催化物种. 反应经历了叔丁醇亚铜与硼烷转金属化、烯烃插入、异萻酸酯插入、与叔丁醇 锂转金属化再生叔丁醇亚铜等步骤. 其中, 异氧酸酯插入为决速步, 经历了一个特殊的三元环过渡态. 如果使用手性 的亚膦酰胺作为配体，则可实现立体选择性的异氧酸酯氢硼乙基化. 对映体选择性控制步为烯烃插入，配体的空间调 控决定了立体选择性. 反应决速步同样是异氧酸酯插入, 但相对使用卡宾配体, 该过程活化能较低. 因此, 亚膦酰胺亚铜催化体系可能具有较高催化活性.
\end{abstract}

关键词＼cjkstart亚铜催化; 密度泛函理论计算; 异氰酸酯; 插入反应

\section{Mechanistic Study of Cu-Catalyzed Addition Reaction of Isocyanates}

\author{
Huang, $\mathrm{Li}^{a} \quad$ Wang, Yuhao $^{a} \quad$ Liu, Jiying ${ }^{a} \quad$ Li, Shijun*a $\quad$ Zhang, Wenjing ${ }^{*, a} \quad$ Lan, Yu ${ }^{*, a, b}$ \\ ( ${ }^{a}$ Green Catalysis Center, College of Chemistry, Zhengzhou University, Zhengzhou 450001) \\ ( ${ }^{b}$ Chongqing Key Laboratory of Theoretical and Computational Chemistry, School of Chemistry and \\ Chemical Engineering, Chongqing University, Chongqing 400001)
}

\begin{abstract}
As a C1 synthetic block, isocyanates were widely used in the synthesis of amide derivatives or heterocyclic compounds. Density functional theory (DFT) calculation was employed to reveal the mechanism of $\mathrm{Cu}(\mathrm{I})$-catalyzed hydroboraethylation of isocyanates. $\mathrm{Cu}(\mathrm{I}) \mathrm{O}^{t} \mathrm{Bu}$ was considered as the active species in catalytic cycle. The catalytic cycle involves transmetallation with borane, alkene insertion, isocyanate insertion, and transmetallation with $\mathrm{LiO}^{t} \mathrm{Bu}$ to yield lithium acetylamide product. The isocyanate insertion was considered as rate-determining step, which underwent a unique threemembered ring type transition state. The catalytic cycle with chiral phosphine ligand was also considered for this reaction. DFT calculation resulted that the enantioselectivity was determined at the alkene insertion step, which was controlled by steric effect of phosphine ligand. Moreover, it was also found that the activation free energy for the rate-determining step with phosphine ligand was lower than that with carbene ligand. Therefore, the phosphine ligand would lead to a higher reaction rate.

Keywords cuprous catalysis; density functional theory (DFT) study; isocyanate; insertion
\end{abstract}

异氧酸酯 ${ }^{[-4}$ - 作为一种含有单一不饱和碳的有机分 子，被广泛应用于合成化学中. 以异氧酸酯作为原料, 通过碳碳键偶联 ${ }^{[-6-6]}$, 可以有效合成碳数加一的羧酸及 酰胺等衍生物. 由于异氰酸酯含有碳氮和碳氧两个不饱 和键, 还可以通过环加成反应构建五元或者六元杂环类 化合物. 异氧酸酯在合成化学上的优异表现, 使其被化 学家们广泛关注.

异氰酸酯的结构特殊性, 使其参与反应时的机理纷
繁复杂. 异氧酸酯可以被看作是二氧化碳的等电子体, 即将二氧化碳中的一个氧原子用亚胺基取代, 就构成了 异氧酸酯. 异氰酸酯的结构中包含了一个累积双键、一 个氧原子和一个亚胺基. 其中碳原子表现为亲电性, 而 氧原子和氮原子表现为亲核性. 因此, 异氧酸酯与亲核 试剂发生反应时, 可经历如 Scheme 1 所示的四种反应 模式. 异㲵酸酯中的碳原子直接与亲核试剂发生加成反 应，构建新的共价键(Scheme 1，a). 反应后得到的阴离

\footnotetext{
* Corresponding authors. E-mail: lishijunzong@zzu.edu.cn; zhangwj@zzu.edu.cn; lanyu@cqu.edu.cn Received July 15, 2021; revised August 15, 2021; published online August 24, 2021.

Project supported by the National Natural Science Foundation of China (No. 21903071) and the Program for Science Technology Innovation Talents in Universities of Henan Province (No. 20HASTIT004).

国家自然科学基金(No. 21903071)和河南省高校科技创新人才支持计划(No. 20HASTIT004)资助项目.
} 
子中间体中, 形式负电荷可被氧原子和氮原子分享, 因 此较为稳定, 可作为活性中间体参与后续反应历程. Tang 课题组 ${ }^{[7]}$ 在之前的理论研究中提出了一种叔胺作 为亲核试剂直接进攻异氧酸酯碳原子的反应机理. Zhang 等 ${ }^{[8]}$ 也报道了烯基碳酸乙烯酯被钯活化得到两性 离子中间体后, 氧负离子作为亲核试剂可直接与异氰酸 酯反应，从而实现分步的 $[3+2]$ 环加成反应. 在这一过 程中, 如果使用 Lewis 酸作为催化剂, 可以有效活化异 氭酸酯的氧原子, 降低反应活化能(Scheme 1, b). D'Elia 和 Poater 等 ${ }^{[9]}$ 在研究糖催化异氰酸酯与氯甲基环氧乙烷 的环加成反应中发现, 糖类催化剂可以与异氧酸酯的氧 原子形成多重氢键, 从而活化异氧酸酯并与亲核性的烷 氧负离子反应. 该路径优于被糖类稳定的烷氧负离子亲 核进攻未活化的异氧酸酯. 过渡金属钉也可以作为 Lewis 酸来活化异㲵酸酯, 实现被外源性的胺亲核进攻 [10]. 当使用金属作为催化剂时, 氮碳双键或氧碳双键均 可插入金属与亲核试剂的共价键中，从而实现异氰酸酯 的加成反应(Scheme 1, c, d). 例如在镁催化异氰酸酯的 嗍氢化一还原反应中, 关键的步骤就是异氰酸酯的碳氧 双键插入镁氢键 ${ }^{[11]}$. 在 Yao 和 Zhao 等 ${ }^{[12]}$ 报道的稀土金 属催化异氰酸酯氢颈基化反应中, 密度泛函理论计算表 明, 反应由异氧酸酯的碳氧双键插入针硫键启动. $\mathrm{O}^{\prime}$ Hair 等 ${ }^{[13]}$ 通过理论计算研究探索了铜催化异氧酸酯 氢芳基化的反应机理. 他们发现, 当芳基铜(I)物种生成 后, 异氰酸酯的碳氧双键插入活化能仅为 $15.4 \mathrm{kcal} / \mathrm{mol}$, 远远低于碳氮双键插入的活化能. 有意思的是, 在锆参 与的异氧酸酯氨基化反应中, Martins 等 ${ }^{[14]}$ 通过结晶和 X 射线衍射分析发现，插入的方式取决于异氰酸酯亚胺基 上的取代基效应. 当取代基为烷基时, 主要发生碳氮双 键插入; 而当取代基为芳基时，则是发生碳氧插入.

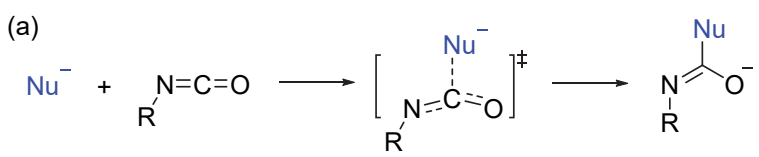

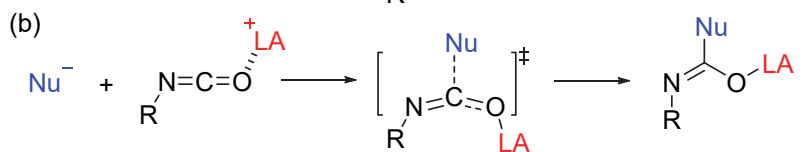

(c)

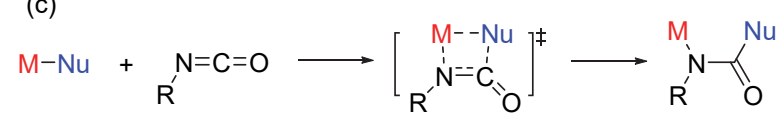

(d)<smiles>[R]N=C(N)O[M]</smiles>

图式 1 异氭酸酯与亲核试剂的反应模式

Scheme 1 Activation models for isocyanates with nucleophiles (a) Nucleophilic attack onto center carbon atom; (b) Nucleophilic attack with the activation of oxygen atom using Lewis acid; (c) $\mathrm{C}=\mathrm{N}$ double bond insertion; (d) $\mathrm{C}=\mathrm{O}$ double bond insertion.

最近, Mazet 等 ${ }^{[15]}$ 报道了一个铜催化异氧酸酯的氢 喼乙基化反应, 实现了较温和条件下的异氧酸酯、烯烃 和频哪醇硼烷三组分的偶联反应(Scheme 2). 无论是使 用卡宾类 ${ }^{[16-17]}$ 配体还是亚膦酰胺类 ${ }^{[18-20]}$ 配体, 都能够高 效率地构建 $\beta$-硼基丙酰胺衍生物 ${ }^{[14,20]}$. 当使用手性亚膦 酰胺类配体时, 可以高选择性地得到 $R$ 型产物. 我们推 测该反应的关键步骤就是异氧酸酯的插入反应. 因此, 我们试图通过密度泛函理论 ${ }^{[21]}$ 探索该反应的机理.

\section{1 结果与讨论}

设想的铜催化异氰酸酯的氢硼乙基化反应相关机 理如 Scheme 3 所示. 氯化亚铜在叔丁醇锂的存在下转 化为叔丁醇亚铜 I 作为活性中间体. 叔丁醇亚铜与嗍烷 发生转金属化反应后得到硼基亚铜中间体 II. 之后的烯
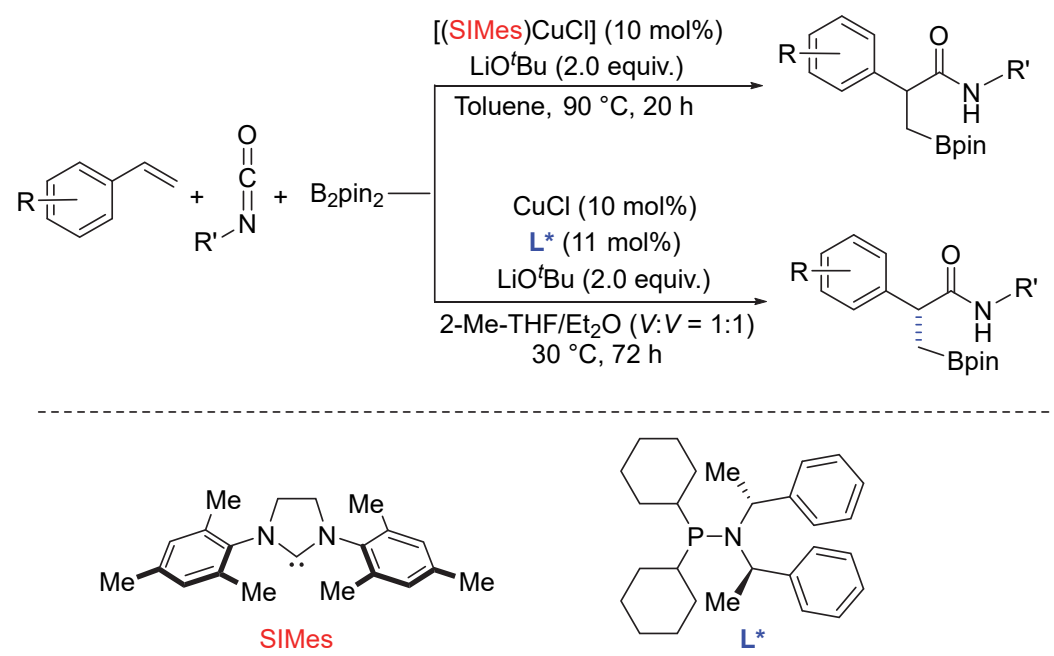

图式 2 铜催化异氧酸酯的氢硼乙基化反应

Scheme $2 \mathrm{Cu}(\mathrm{I})$-catalyzed hydroboraethylation of isocyanates 
烃插入得到苄基亚铜中间体 IV. 当异氰酸酯进入反应 体系后，碳氮双键可插入碳铜键后得到酰胺基亚铜中间 体 VI, 并与叔丁醇锂发生转金属化释放产物 VII 后再生 叔丁醇亚铜中间体 $\mathbf{I}$ 完成催化循环. 为了进一步探索该 反应的机理, 我们使用密度泛函理论计算对该反应途径 进行了研究.

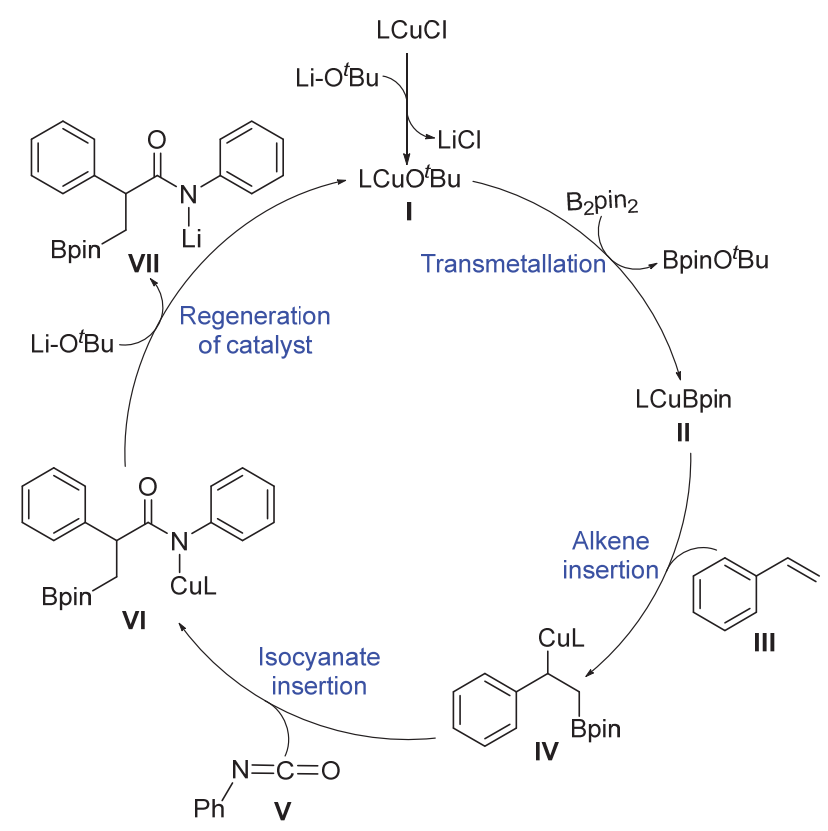

图式 3 铜催化异氰酸酯的氢嗍乙基化反应机理

Scheme 3 General mechanism of $\mathrm{Cu}(\mathrm{I})$-catalyzed hydroboraethylation of isocyanates.

铜催化异氧酸酯的氢嗍乙基化反应中, 催化剂预活 化的反应势能面如图 1 所示. 我们使用卡宾络合的氯化 亚铜 1 作为催化剂前体, 它可以与叔丁醇锂结合得到复 合物 2 并吸热 $0.6 \mathrm{kcal} / \mathrm{mol}$. 经过一个活化能仅为 1.8 $\mathrm{kcal} / \mathrm{mol}$ 的过渡态 3-ts 后, 发生转金属化反应, 并释放 氯化锂得到叔丁醇亚铜中间体 $\mathbf{5}$. 这一过程整体放热为 $7.8 \mathrm{kcal} / \mathrm{mol}$.
计算表明, 相对于氯化亚铜来说, 活性催化物种叔 丁醇亚铜 5 的生成是一个放热过程. 接下来，以该物种 作为起点计算了整个催化循环的势能面(图 2). 叔丁醇 亚铜 5 可以与嗍烷络合生成配合物 6, 之后经历过渡态 7-ts 发生转金属化反应。该过程整体活化能为 18.4 $\mathrm{kcal} / \mathrm{mol}$. 释放掉硼酸酯后，得到硼基亚铜中间体 8. 该 中间体可与苯乙烯配位, 得到中间体 9. 计算表明, 后 续的烯烃插入可经历四元环过渡态 10-ts, 得到茮基亚 铜中间体 11, 该过程放热为 $27.7 \mathrm{kcal} / \mathrm{mol}$. 我们使用异 氯酸苯酯作为模型反应物进行理论计算. 有意思的是, 由于亲核的烷基在与异氰酸酯反应时并未从铜上脱离, 因此后续亲核进攻经历三元环过渡态 12-ts 实现. 过渡 态 12-ts 的结构如图 2 所示. 将要成键的碳碳距离为 $0.222 \mathrm{~nm}$, 将要断裂的碳铜距离为 $0.208 \mathrm{~nm}$. 而这时铜 与异咨酸酯的碳原子距离也仅为 $0.209 \mathrm{~nm}$, 说明它们之 间也有着较强的作用. 额外的分子中的原子 (atoms-inmolecules, AIM)计算表明, 铜与异氧酸酯的碳原子之间 关键键点(bond critical point, BCP) 位置电子密度为 0.14 a.u., 表明它们之间有较强的作用. 对过渡态 12-ts 做内 禀反应坐标分析表明，经过该过渡态后，将会得到酰胺 基亚铜 13 中间体. 碳氮双键经历过渡态 12-ts 插入的活 化能为 $25.4 \mathrm{kcal} / \mathrm{mol}$. 得到的酰胺基亚铜 13 可与叔丁醇 锂发生转金属化反应，释放酰胺基锂后再生叔丁醇亚铜 中间体 5. 计算表明，异氧酸酯插入这一步为整个反应 的决速步.

为了进一步探索配体对该反应的影响, 我们还计算 了使用手性亚膦酰胺作为配体时的催化循环势能面(图 3). 与使用卡宾配体类似, 该反应势能面同样以亚膦酰 胺配合的叔丁醇亚铜中间体 15 作为起点. 该物种与嗍 烷经历过渡态 16-ts 发生转金属化反应后得到相应的硼 基亚铜中间体 17. 当使用手性的亚膦酰胺作为配体时,

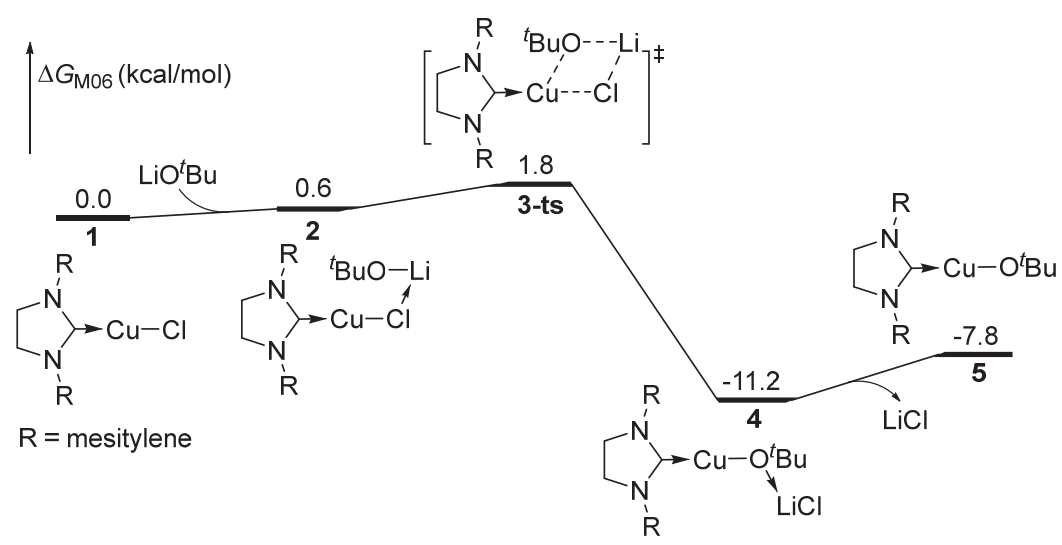

图 1 亚铜催化剂预活化路径的势能面

Figure 1 Free energy profiles for the pre-activation of $\mathrm{Cu}(\mathrm{I})$ species 


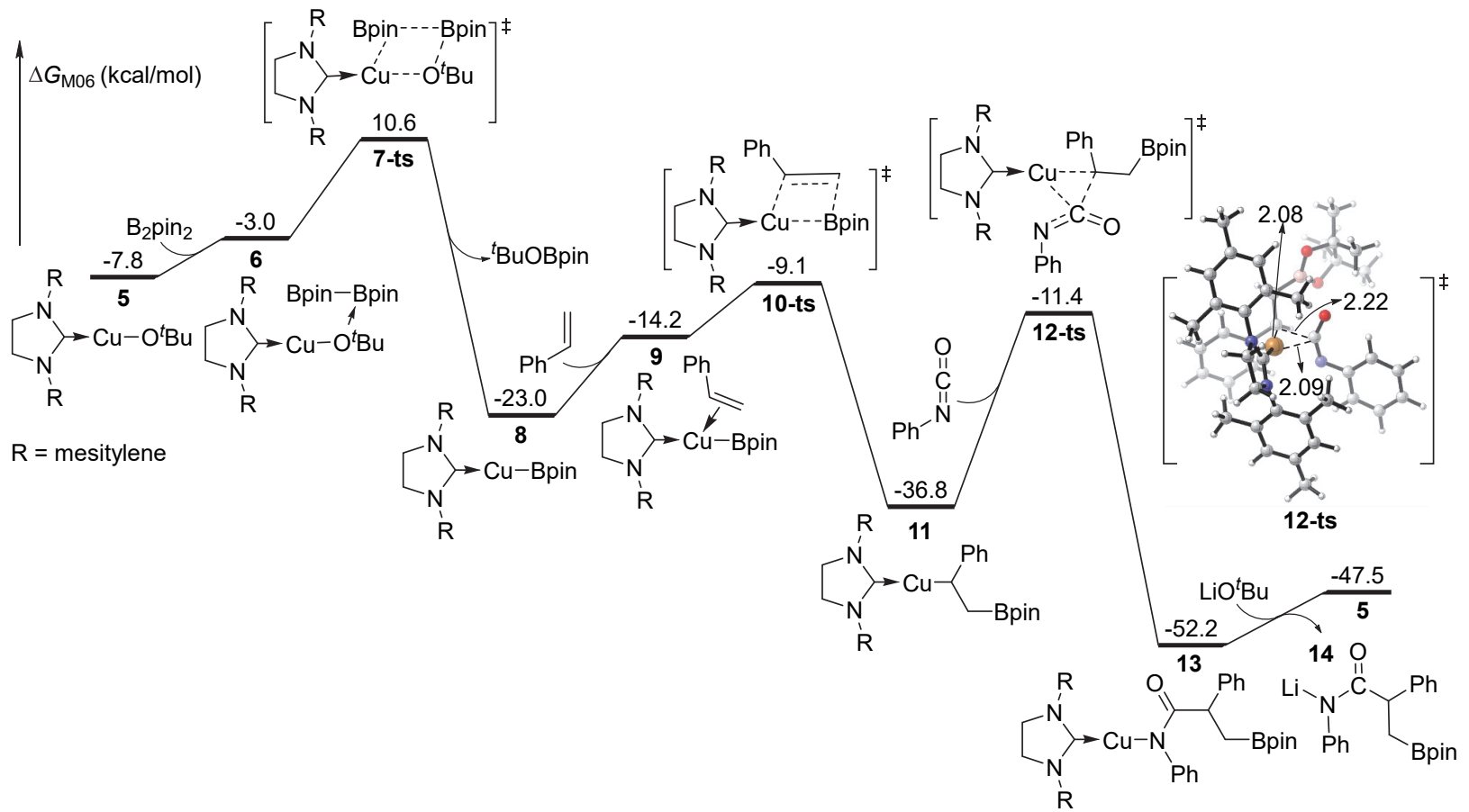

图 2 卡宾亚铜催化异氰酸酯氢嗍乙基化反应催化循环的势能面

Figure 2 Free energy profiles for the catalytic cycle of $\mathrm{Cu}(\mathrm{I})$-carbene-catalyzed hydroboraethylation of isocyanates Bond lengths in angstrom
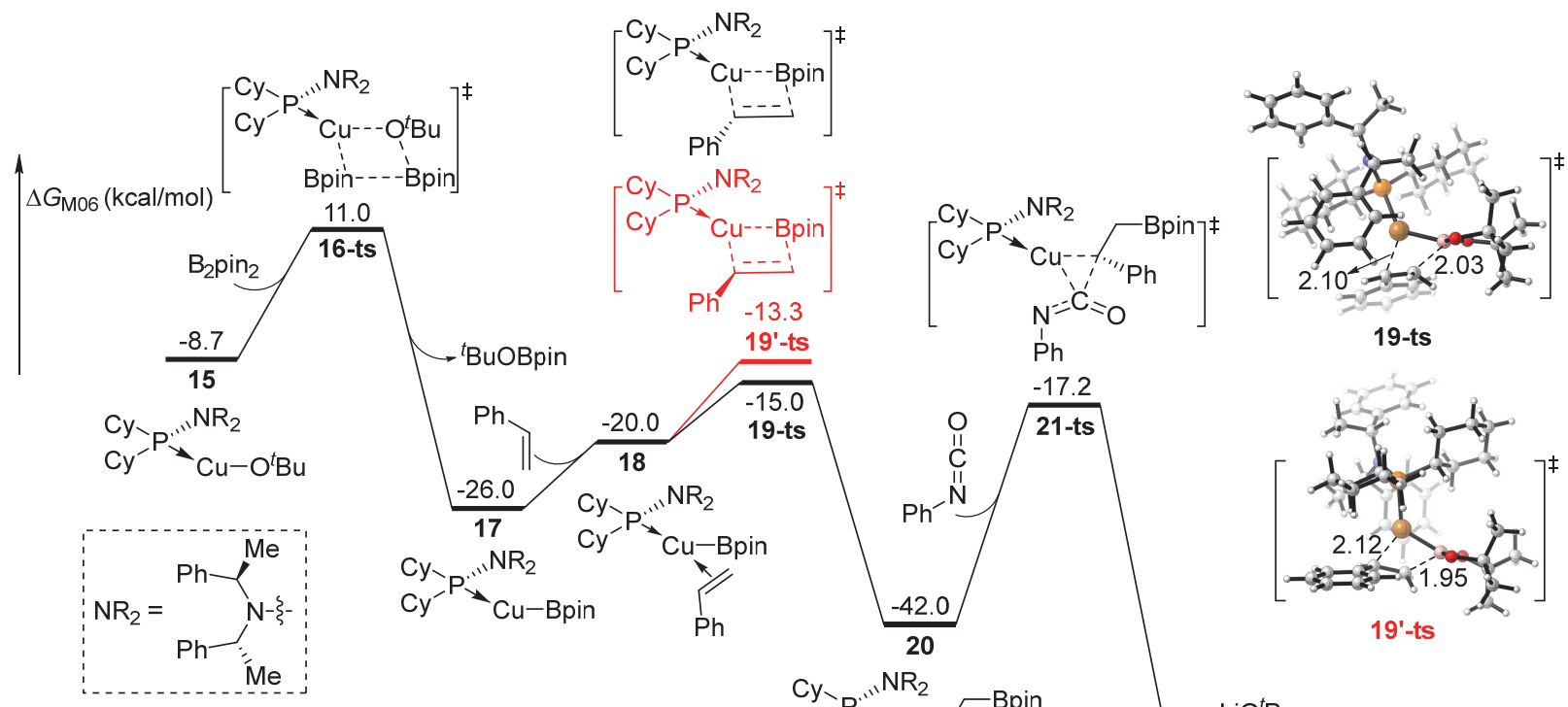

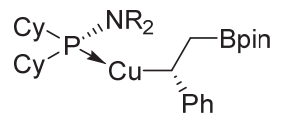
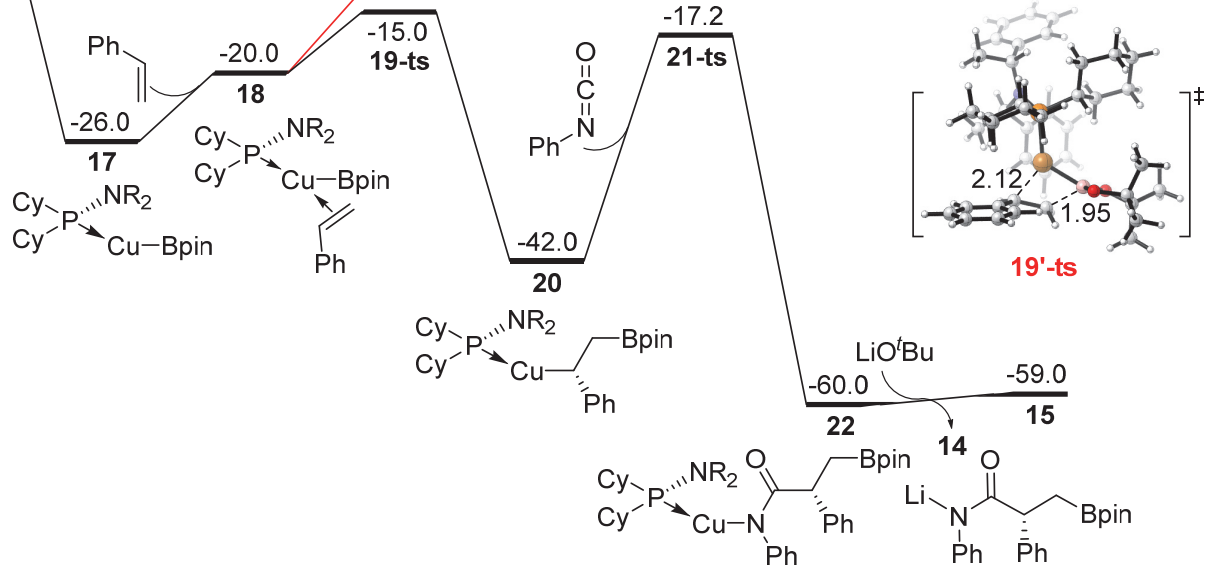

图 3 膦-亚铜催化异㲵酸酯氢硼乙基化反应催化循环的势能面

Figure 6 Free energy profiles for the catalytic cycle of $\mathrm{Cu}(\mathrm{I})$-phosphine-catalyzed hydroboraethylation of isocyanates Bond lengths in angstrom

苯乙烯的插入有两种方式: 经历过渡态 19-ts, 苯乙烯的 $R e$-面发生插入; 或经历过渡态 19'-ts, 苯乙烯的 $S i$-面发 生插入. 计算表明 19-ts 的能量比 19'-ts 低 $1.7 \mathrm{kcal} / \mathrm{mol}$.
这说明苯乙烯 $R e$-面插入为优势反应路径，这样可得到 $R$-构型为主的产物, 与实验观测一致. 过渡态 19-ts 和 19'-ts 的结构如图 3 所示，可以清晰看出，苯乙烯的苯基 
在 19'-ts 中与配体距离较近, 有较大排斥力, 这应该就 是立体选择性产生的原因. 当苯乙烯插入并得到相应的 苄基亚铜 20 后, 异氰酸酯的碳氮双键可以经历过渡态 21-ts 插入到碳铜键中. 计算表明, 这一步的活化能为 $24.8 \mathrm{kcal} / \mathrm{mol}$, 相比使用卡宾配体的催化循环中对应的 步骤, 活化能低 $0.6 \mathrm{kcal} / \mathrm{mol}$. 这说明, 手性亚磷酰胺配 体可能有利于该反应进行.

\section{2 结论}

通过密度泛函理论计算研究了亚铜催化异氧酸酯 氢嗍乙基化反应的机理. 研究结果表明, 该反应经历了 叔丁醇亚铜与硼烷转金属化、烯烃插入、异氧酸酯插入 以及与叔丁醇锂转金属化再生叔丁醇亚铜等步骤. 其 中, 异氰酸酯经历一个三元环过渡态插入铜碳键为决速 步. 如果使用手性的亚膦酰胺作为配体, 则可实现立体 选择性的异氰酸酯氢硼乙基化. 对映体选择性控制步为 烯烃插入, 配体的空间调控决定了对映体选择性. 此外, 计算表明, 使用亚膦酰胺作为配体, 反应决速步同样是 异氰酸酯插入, 但相对使用卡宾配体, 该过程活化能较 低. 因此, 亚膦酰胺-亚铜催化体系可能具有较高催化 活性.

\section{3 计算细节}

所有计算均在 Gaussian $09^{[22]}$ 计算软件下执行. 理 论计算中涉及的反应物、过渡态、中间体及产物均采用 B3LYP ${ }^{[23]}$ 密度泛函方法进行结构优化. 其中, $\mathrm{Cu}$ 原子采 用 $\mathrm{SDD}^{[24]}$ 噟势基组, 其它原子采用 $6-31 \mathrm{G}(\mathrm{d})^{[25]}$ 基组. 对 于卡宾-亚铜体系, 所有过渡态和中间体的优化都在甲 苯溶剂中进行; 对于亚膦酰胺-亚铜催化体系, 所有过 渡态和中间体的优化都在乙醚与 2-甲基-四氢呋喃的混 合溶剂(介电常数为 $5.3 \mathrm{~F} / \mathrm{m}$, 由两种溶剂的介电常数按 照 $1: 1$ 权重计算得出)中进行. 两种情况均使用

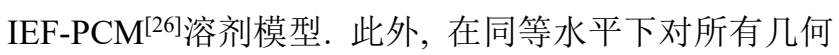
结构进行振动频率分析以确保所有的反应物、产物和中 间体没有虚频, 所有过渡态均有且只有一个虚频. 为了 验证过渡态的合理性, 通过内禀反应坐标(IRC) ${ }^{[27]}$ 在同 样水平下的计算证实每个过渡态均能正确连接相关的 反应物和产物.

最后, 基于优化后的几何结构, 对所有驻点的能量 在 M06 ${ }^{[28]} / \mathrm{def} 2-\mathrm{TZVP}{ }^{[29]} / \mathrm{IEF}-\mathrm{PCM}$ 水平进行了单点校正. 我们选择使用 M06 泛函进行单点能校正的原因有两个: (1) B3LYP 与 M06 泛函位于 Jacob's Ladder 的同一层, 同 属于杂化泛函, 但 B3LYP 属于 hybrid GGA, M06 泛函属 于 hybrid meta-GGA, 较前者添加了动能密度项(kinetic density $\tau$ ), 理论预测精度更高, 且 M06 泛函对色散作用
的计算结果更好; (2) B3LYP 与 M06 泛函结合使用研究 反应机理已被广泛使用 ${ }^{[30]}$, 其可靠性已得到证实.

文中涉及的所有吉布斯自由能均是 M06/def2TZVP/IEF-PCM 水平计算的电子能与 B3LYP/6-31G(d)/ SDD/IEF-PCM 方法下计算的吉布斯矫正值能之和. 关 键过渡态的几何构型由 CYLview ${ }^{[31]}$ 软件绘制.

辅助材料(Supporting Information) 所有结构的能量 值和原子坐标. 这些材料可以免费从本刊网站(http:// sioc-journal.cn/)上下载.

\section{References}

[1] Monie, F.; Grignard, B.; Thomassin, J.-M.; Mereau, R.; Tassaing, T.; Jerome, C.; Detrembleur, C. Angew. Chem., Int. Ed. 2020, 59, 17033.

[2] Allen, M. A.; Ivanovich, R. A.; Beauchemin, A. M. Angew. Chem., Int. Ed. 2020, 59, 23188.

[3] Sharpe, H. R.; Geer, A. M.; Lewis, W.; Blake, A. J.; Kays, D. L. Angew. Chem., Int. Ed. 2017, 56, 4845.

[4] Zheng, S.; Primer, D. N.; Molander, G. A. ACS Catal. 2017, 7, 7957.

[5] Mohjer, F.; Mofatehnia, P.; Rangraz, Y.; Heravi, M. M. J. Organomet. Chem. 2021, 936, 121712.

[6] Cui, C.-X.; Chen, H.; Li, S.-J.; Zhang, T.; Qu, L.-B.; Lan, Y. Coord. Chem. Rev. 2020, 412, 213251.

[7] Zhang, C.; Zhu, Y. Y.; Wei, D. H.; Sun, D. Z.; Zhang, W. J.; Tang, M. S. J. Phys. Chem. A 2010, 114, 2913.

[8] Khan, A.; Xing, J. X.; Zhao, J. M.; Kan, Y. H.; Zhang, W. B.; Zhang, Y. J. Chem.-Eur. J. 2015, 21, 120.

[9] Yingcharoen, P.; Natongchai, W.; Poater, A.; D'Elia, V. Catal. Sci. Technol. 2020, 10, 5544.

[10] Bruffaerts, J.; von Wolff, N.; Diskin-Posner, Y.; Ben-David, Y.; Milstein, D. J. Am. Chem. Soc. 2019, 141, 16486.

[11] Yang, Y.; Anker, M. D.; Fang, J.; Mahon, M. F.; Maron, L.; Weetman, C.; Hill, M. S. Chem. Sci. 2017, 8, 3529.

[12] Lu, C. R.; Hu, L. J.; Zhao, B.; Yao, Y. M. Organometallics 2019, $38,2167$.

[13] Yang, Y.; Canty, A. J.; O'Hair, R. A. J. J. Mass Spectrom. 2021, 56, e4579.

[14] Alves, L. G.; Madeira, F.; Munhá, R. F.; Barros, S.; Veiros, L. F.; Martins, A. M. Dalton Trans. 2015, 44, 1441.

[15] Fiorito, D.; Liu, Y.; Besnard, C.; Mazet, C. J. Am. Chem. Soc. 2020, $142,623$.

[16] Peris, E. Chem. Rev. 2018, 118, 9988.

[17] Kuwata, S.; Hahn, F. E. Chem. Rev. 2018, 118, 9642.

[18] Xu, G. Q.; Senanayake, C. H.; Tang, W. J. Acc. Chem. Res. 2019, $52,1101$.

[19] Fu, W. Z.; Tang, W. J. ACS Catal. 2016, 6, 4814.

[20] de Figueiredo, R. M.; Suppo, J.-S.; Campagne, J.-M. Chem. Rev. 2016, 116, 12029.

[21] (a) Kohn, W.; Sham, L. J. Phys. Rev. 1965, 140, A1133. (b) Kohn, W. Rev. Mod. Phys. 1999, 71, 1253.

[22] Frisch, M. J.; Trucks, G. W.; Schlegel, H. B.; Scuseria, G. E.; Robb, M. A.; Cheeseman, J. R.; Scalmani, G.; Barone, V.; Mennucci, B.; Petersson, G. A.; Nakatsuji, H.; Caricato, M.; Li, X.; Hratchian, H. P.; Izmaylov, A. F.; Bloino, J.; Zheng, G.; Sonnenberg, J. L.; Hada, M.; Ehara, M.; Toyota, K.; Fukuda, R.; Hasegawa, J.; Ishida, M.; Nakajima, T.; Honda, Y.; Kitao, O.; Nakai, H.; Vreven, T.; Montgomery, Jr., J. A.; Peralta, J. E.; Ogliaro, F.; Bearpark, M.; Heyd, J. J.; Brothers, E.; Kudin, K. N.; Staroverov, V. N.; Kobayashi, R.; Normand, J.; Raghavachari, K.; Rendell, A.; Burant, J. C.; Iyengar, S. S.; Tomasi, J.; Cossi, M.; Rega, N.; Millam, N. J.; Klene, M.; 
Knox, J. E.; Cross, J. B.; Bakken, V.; Adamo, C.; Jaramillo, J.; Gomperts, R.; Stratmann, R. E.; Yazyev, O.; Austin, A. J.; Cammi, R.; Pomelli, C.; Ochterski, J. W.; Martin, R. L.; Morokuma, K.; Zakrzewski, V. G.; Voth, G. A.; Salvador, P.; Dannenberg, J. J.; Dapprich, S.; Daniels, A. D.; Farkas, Ö.; Foresman, J. B.; Ortiz, J. V.; Cioslowski, J.; Fox, D. J. Gaussian 09, Revision D.01, Gaussian, Inc., Wallingford, CT, 2013.

[23] (a) Becke, A. D. J. Chem. Phys. 1993, 98, 5648.

(b) Stephens, P. J.; Devlin, F. J.; Chabalowski, C. F.; Frisch, M. J. J. Phys. Chem. 1994, 98, 11623.

(c) Lee, C.; Yang, W.; Parr, R. G. Phys. Rev. B: Condens. Matter Mater. Phys. 1988, 37, 785.

[24] (a) Dolg, M.; Stoll, H.; Preuss, H. J. Chem. Phys. 1989, 90, 1730.

(b) Dolg, M.; Wedig, U.; Stoll, H.; Preuss, H. J. Chem. Phys. 1987, 86,866 .

[25] (a) Francl, M. M. J. Chem. Phys. 1982, 77, 3654

(b) Hehre, W. J. J. Chem. Phys. 1972, 56, 2257.

(c) Hariharan, P. C.; Pople, J. A. Theor. Chim. Acta 1973, 28, 213.
[26] (a) Sang-Aroon, W.; Ruangpornvisuti, V. Int. J. Quantum Chem. 2008, $108,1181$.

(b) Tomasi, J.; Mennucci, B.; Cancès, E. J. Mol. Struct. 1999, 464 , 211.

[27] (a) Gonzalez, C.; Schlegel, H. B. J. Chem. Phys. 1989, 90, 2154. (b) Gonzalez, C.; Schlegel, H. B. J. Phys. Chem. 1990, 94, 5523.

[28] Zhao, Y.; Truhlar, D. Theor. Chem. Acc. 2008, 120, 215.

[29] (a) Weigend, F.; Ahlrichs, R. Phys. Chem. Chem. Phys. 2005, 7, 3297.

(b) Cancès, E.; Mennucci, B.; Tomasi, J. J. Chem. Phys. 1997, 107, 3032.

[30] (a) Liu, C.; Li, S.-J.; Han, P.; Qu, L.-B.; Lan, Y. Mol. Catal. 2021, 499,111318

(b) Shen, B.; Liu, S.; Zhu, L.; Zhong, K.; Liu, F.; Chen, H.; Bai, R.; Lan, Y. Organometallics, 2020, 39, 2813.

(c) Wang, Y.; Liao, W.; Huang, G.; Xia, Y.; Yu, Z.-X. J. Org. Chem. 2014, 79, 5684 . 